\title{
DIRECT-COOLING MA CAVITY FOR J-PARC SYNCHROTRONS
}

\author{
T. Uesugi*, Y. Mori, C. Ohmori, A. Takagi, M. Yoshii, KEK, Tsukuba, Japan \\ M. Yamamoto, F. Tamura, JAERI, Tokai, Japan \\ and A. Schnase, COSY, Juelich, Germany
}

\begin{abstract}
The first rf cavity for the J-PARC(Japan Proton Accelerator Research Complex) $50 \mathrm{GeV}$ synchrotron has been developed. In the cavity, magnetic-alloy(MA) cores are cooled by water directly. With the cavity, we have accomplished an acceleration field of more than $50 \mathrm{kV}$ in $1.776 \mathrm{~m}$ cavity length. We show the design of the rf cavity and results of high-power tests.
\end{abstract}

\section{INTRODUCTION}

In the J-PARC project[1], a proton beam will be accelerated from $181 \mathrm{MeV}$ to $3 \mathrm{GeV}$ in the rapid cycle synchrotron(RCS), and then up to $50 \mathrm{GeV}$ in the main ring(MR). The parameters of the synchrotrons are listed in Table 1. In both synchrotrons, an accelerating field of about $30 \mathrm{kV} / \mathrm{m}$ is required. In order to realize such a high field-gradient, we will employ rf cavities loaded with magnetic-alloy(MA) cores whose impedance is kept high under high magnetic field as $2 \mathrm{kG}$ at least[2]. The average power-dissipation of the cavity, taking the duty factor into account, will become about $120 \mathrm{~kW} / \mathrm{cav}(40 \mathrm{~kW} /$ cell) for the RCS and about $240 \mathrm{~kW} / \mathrm{cav}(80 \mathrm{~kW} / \mathrm{cell})$ for the MR, respectively.

Because of the high power-dissipation, it is important to cool the cores efficiently. We have developed two types of rf cavities as candidates for the J-PARC synchrotrons; One is indirect-cooling MA cavity, and the other is directcooling one. In the indirect-cooling cavity, the MA cores are cooled by cooling-disks indirectly[3]. With the cavity a gap voltage of $10 \mathrm{kV}$ was stably obtained in the RCS

Table 1: Rf parameters of the J-PARC synchrotrons.

\begin{tabular}{|l|l|l|}
\hline parameter & RCS & MR \\
\hline circumference & $348.3 \mathrm{~m}$ & $1567.9 \mathrm{~m}$ \\
particle energy & $0.181 \sim 3 \mathrm{GeV}$ & $3 \sim 50 \mathrm{GeV}$ \\
revolution freq. & $0.94 \sim 1.67 \mathrm{MHz}$ & $1.67 \sim 1.72 \mathrm{MHz}$ \\
repetition time & $40 \mathrm{msec}$ & $\sim 3 \mathrm{sec}$ \\
harmonic no. & 2 & 9 \\
\hline max. rf-voltage & $450 \mathrm{kV}$ & $280 \mathrm{kV}$ \\
duty(power) & $\sim 30 \%$ & $\sim 60 \%$ \\
no. of cavities & 11 & 6 \\
cavity length & $1.776 \mathrm{~m}$ & $1.776 \mathrm{~m}$ \\
quality factor & $2 \sim 3$ & $\sim 10$ \\
no. of gaps & $3 /$ cavity & $3 /$ cavity \\
\hline
\end{tabular}

\footnotetext{
${ }^{*}$ He has moved to NIRS, Inage-ku, Chiba, Japan
}

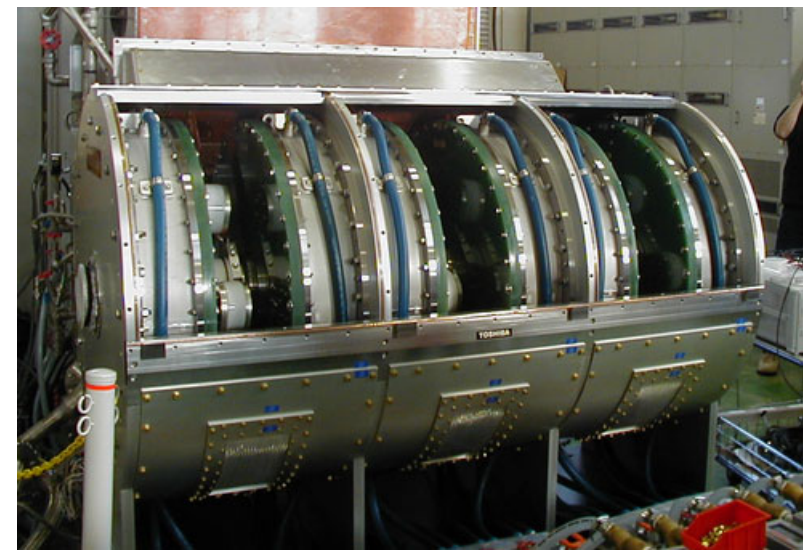

Figure 1: Direct-cooling MA cavity.

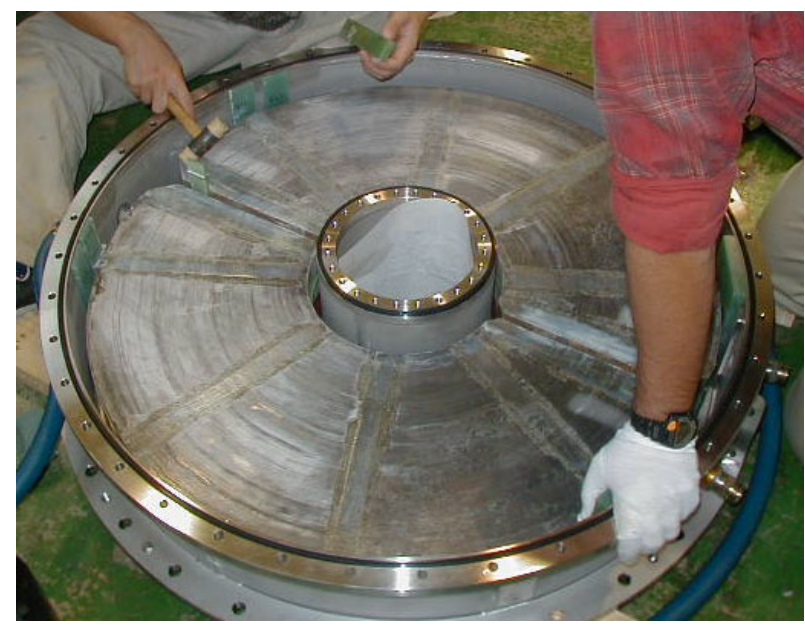

Figure 2: Inside the tank.

frequency-band, and the averaged-power of $36 \mathrm{~kW} / \mathrm{gap}$ was removed by water.

In the direct-cooling cavity, on the other hand, the MA cores are installed in a water-sealed tank and cooled by water directly. This type of cavity is used in the NIRSHIMAC[5] and in the KEK-PS second-harmonic cavity[6]. In this paper, we report the design and the performances of the direct-cooling MA cavity for the J-PARC synchrotrons.

\section{CAVITY DESIGN}

Figure 1 shows the picture of the direct-cooling MA cavity. The cavity consists of three identical cells, and each cell has an accelerating gap at the center, three MA cores on both side, and vacuum capacitors for tuning. It has no dynamic tuning system because of the broad-band characteristics of the MA. The accelerating gaps are connected 
with a couple of bus-bars in parallel. The rf power is put by a 1 MW push-pull amplifier with two tetrodes(TH558)[4].

The dimension of the MA cores is $800 \mathrm{~mm}(\mathrm{OD})$ $250 \mathrm{~mm}$ (ID)-35 $\mathrm{mm}$ (TH). The cores are diametrically cut in two by water-jet-cut in order to control the quality factor[5]. The accuracy of the cut-surface is $\sim 1 \mathrm{~mm}$, which should be improved not to disturb the water-flow there. Three MA cores are piled in a water-sealed tank with FRPdisks inserted between, and fixed by spacers at outer side and the cut-surfaces of the cores(Fig. 2). Those spacers are not only for the core-support but also for excluding a part of water around the core, which causes the impedance reduction due to the dielectric effect of the water $(\varepsilon \sim 80)$. The band-width, or quality factor $(Q)$ of the cavity in other word, is controlled by changing the thickness of the spacers at the cut-surface; It is $1 \mathrm{~mm}$ for the $\operatorname{RCS}(Q \sim 2)$ and $10 \mathrm{~mm}$ for the $\operatorname{MR}(Q \sim 10)$.

Cooling-water comes from the bottom of the tank and flows upward. The configuration of the core-support pieces on the FRP-disks should be optimized to diffuse the waterflow. In a direct-cooling MA cavity, it is important to protect the cores from rusting or corrosion. We are planning to protect the cores by coating them with epoxy- and then polyester-resin with $\sim 0.2 \mathrm{~mm}$ thickness. According to a small-sample test, the surface of an MA core has survived in a $6 \% \mathrm{FeCl}_{3}$ solution at $50{ }^{\circ} \mathrm{C}$ over 24 hours.

\section{HIGH-POWER TEST}

High-power tests of the cavity were carried out with the RCS and the MR setup, respectively.

\section{1 cavity impedance}

As is written before, the resonant frequency and the band-width of the cavity are determined by the tuning capacitance and gap width of the cut-core. The parameters of the cavity are shown in Table 2. Figure 3 shows the impedance of the cavity measured from the accelerating gap directly. The effects of the capacitance due to the tube and the water are included. In the Fig. 3, the dashed line shows the case where three cells are filled with the cores and connected to the amplifier, and rigid ones show only one cell is connected. Three cells impedance for the RCS

Table 2: Parameters of the cavity for the RCS and the MR setup.

\begin{tabular}{|l|l|l|l|}
\hline parameter & RCS & \multicolumn{2}{|c|}{ MR } \\
\hline $\begin{array}{l}\text { cut-core gap width } \\
\text { tuning capacitance }\end{array}$ & $1.5 \mathrm{~mm}$ & \multicolumn{2}{|c|}{$10 \mathrm{~mm}$} \\
\hline number of cells & $100 \mathrm{pF}$ & \multicolumn{2}{|c|}{$1600 \mathrm{pF}$} \\
peak impedance & $837 \Omega$ & 1 & 3 \\
resonant frequency & $1605 \mathrm{kHz}$ & $186 \Omega$ & $263 \Omega$ \\
band-FWHM & $549 \mathrm{kHz}$ & $189 \mathrm{kHz}$ & $1821 \mathrm{kHz}$ \\
\hline
\end{tabular}

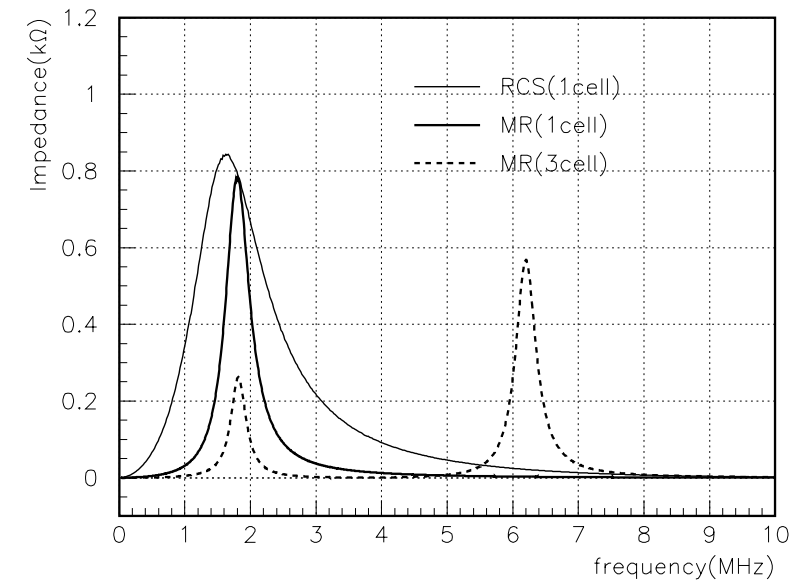

Figure 3: Cavity impedance measured from the gap.

setup is not measured in this moment. Without the water inside the tank, one-cell impedance with the MR setup was $960 \Omega$ at its peak; The impedance reduction due to the water is about $18 \%$.

As shown in Fig. 3, the band-width with the RCS setup was not enough to cover the required range, because the cut-surfaces of the cores were not flat enough to reduce the Q-value.

There is a parasitic resonance at $6.2 \mathrm{MHz}$ when three cells are loaded. That resonance came from the inductance of the bus-bars and resonant capacitors, and it was found that we could damp it by putting damping resistors on the bus-bar and adding another bus-bar[7]. In addition, another impedance measurement with a conductor wire showed that the parasitic resonance is not observed[8].

\subsection{MR setup}

We have input an rf-power into just one cell of the cavity with the MR setup. The operating frequency was fixed at $1.785 \mathrm{MHz}$. A gap voltage of $18 \mathrm{kV}$ was successfully obtained with $50 \%$ duty in $2.0 \mathrm{sec}$ on/off period. The averaged rf-power was calculated to be $103 \mathrm{~kW}$, which was beyond the requirement. The wave-form of the gap voltage looked quite good. The temperature rise of the cooling-water, whose flux was $50 \mathrm{l} / \mathrm{min}$ for each tank, was about $15{ }^{\circ} \mathrm{C}$; That value was consistent with the powerdissipation at the cores.

In the next, we have input power into the whole cavity, ie. three-cells cavity. At $1.81 \mathrm{MHz}$ the voltage up to $18 \mathrm{kV} /$ gap was stably obtained for 6 hours. After a 49 hours long run with $17 \mathrm{kV} / \mathrm{gap}$, we opened the water-tanks of the cavity and found no damage on the cores or FRP pieces. The power-test is limited by the water-cooling system of the experimental facility. Thus, the direct-cooling MA cavity has completely satisfied the requirements of highvoltage and high-power performance for the MR. 


\subsection{RCS setup}

Preliminary high-power test for the RCS setup has also been done with the cavity. The rf-power was continuously input into one cell of the cavity. The gap voltage of $12.4 \mathrm{kV}$ was obtained; That corresponds to the rf-power of $90 \mathrm{~kW}$, which is more than twice of the requirement. The temperature rise of the water was $12{ }^{\circ} \mathrm{C}$.

After 5 hours high-power operation, a part of the cores were damaged by heat at the cut-surface where the heat was concentrated. Because the cores were designed for the MR, the flatness of the cut-surface was not enough and and the coolant is difficult to flow in the narrow-gap case. We are trying to improve the water-flow control by improving the smoothness of the cut-surface.

\section{COMPARISON TO THE INDIRECT-COOLING}

We compare the direct- and indirect-cooling[3] MA cavities at this moment. With the indirect-cooling cavity with the RCS setup, an rf power of $40 \mathrm{~kW} /$ cell was succesfully cooled by water. On the other hand, high-power test has not been tried with the MR setup because of the heat concentration at the outside of the MR cores, which was observed in a single-core test in wide gap $(\sim 1 \mathrm{~cm})$ condition. In addition, the adhering strength of the insulator between a core and a cooling-disk is important, because a presence of an air-gap between them seriously reduces the local cooling efficiency. A part of the insulators was peeled off by thermal stress under a high-power operation. The cooling efficiency of the direct cooling is better than the indirect one. This is the main reason we decide to use the direct cooling scheme for J-PARC cavity.

The impedance reduction comes from the capacitive effects between cores and other material. It is larger in case of the direct-cooling than that of indirect one because of the high dielectric constant of water. The effect becomes severe at the high frequency region. To reduce the effect, the purity of water is important. It is possible to overcome the disadvantage by using a coolant with lower dielectric constant like fluorinert[6].

There might be a situation where one have to change the gap-distance slightly to readjust the bandwidth of the cavity. In the indirect-cooling cavity, it is easy to push one half of the MA cores to control the gap-distance. However, in the direct-cooling one, we need to open the tanks and fix the cores with a different thickness of the spacers. We need to improve the maintenanceability of the direct-cooling cavity.

\section{SUMMARY}

We have newly developed a direct-cooling MA cavity for the J-PARC synchrotrons, and examined it with highpower $\mathrm{rf}$. The cavity accomplished the required rf-voltages for the MR and the RCS operations. The cores were cooled well with the MR setup. However, it was not sufficient with the RCS setup with the heavy duty operation( $200 \%$ of the requirement) where the gap distance was narrower. This problem will be solved by improvement of the cut-surface.

\section{REFERENCES}

[1] Accelerator group, JAERI/KEK Joint Project Team, 'Accelerator Technical Design Report for High-Intensity Proton Accelerator Facility Project', KEK-report.

[2] Y. Tanabe et al., EVALUATION OF MAGNETIC ALLOYS FOR JHF RF CAVITY, APAC98, Tsukuba, 1998.

[3] C. Ohmori et al., HIGH FIELD GRADIENT CAVITY FOR JAERI-KEK JOINT PROJECT, EPAC2002, Paris, 2002.

[4] M. Yoshii et al., RF Acceleration Systems for the JAERIKEK Joint Project, EPAC2002, Paris, 2002.

[5] C. Ohmori et al., High Field-Gradient Cavities Loaded with Magnetic Alloys for Synchrotrons, PAC1999, New York, 1999.

[6] M. Yoshii et al., MA RF Cavity for the KEK $12 \mathrm{GeV}$ PS, EPAC2000, Stockholm, 2000.

[7] M. Brennan, The Upgraded RF System for the AGS and High Intensity Proton Beams, Dallas, PAC95, 1995.

[8] T. Toyama, private communication. 